\title{
Cytotoxicity of Secondary Metabolites from Dracaena viridiflora Engl \& Krause and their Semisynthetic Analogues
}

\author{
Rémy Bertrand Teponno ${ }^{* 1,2}$, Jean Paul Dzoyem ${ }^{2,4}$, Raymond Ngansop \\ Nono $^{1}$, Ulrich Kauhl ${ }^{2}$, Louis P. Sandjo ${ }^{2}$, Léon Azefack Tapondjou ${ }^{1}$, \\ Udo Bakowsky ${ }^{4}$ and Till Opatz ${ }^{* 2}$ \\ ${ }^{I}$ Department of Chemistry, Faculty of Science, University of Dschang, Box 67, Dschang, Cameroon \\ ${ }^{2}$ Johannes Gutenberg University Mainz, Institute of Organic Chemistry, Duesbergweg 10-14, D- \\ 55128 Mainz, Germany \\ ${ }^{3}$ Department of Biochemistry, Faculty of Science, University of Dschang, Box 67, Dschang, Cameroon \\ ${ }^{4}$ Department of Pharmaceutics and Biopharmaceutics, Philipps University of Marburg, Robert-Koch- \\ Str.4, D-35037 Marburg, Germany
}

(Received March 04, 2017; Revised May 04, 2017; Accepted May 12, 2017)

\begin{abstract}
The $\mathrm{MeOH}$ extract of Dracaena viridiflora was found to display significant cytotoxicity against some cancer cell lines. Systematic phytochemical investigation of this extract led to the isolation and structure elucidation of ten secondary metabolites including five spirostane (1-5) and one furostane (6) steroidal saponins. Furthermore, some acetylated spirostane analogues and three previously unreported derivatives with the $22,26-$ epoxycholesta-5,22-diene skeleton (15-17) were prepared from trillin (1), prosapogenin A of dioscin (2) and dioscin (4) by reaction with $\mathrm{ZnCl}_{2} / \mathrm{Ac}_{2} \mathrm{O}$. Among the isolated and semisynthetic compounds, dioscin showed the most potent cytotoxicity against A549, Jurkat and Skov-3 cells with $\mathrm{IC}_{50}$ values of $0.42,1.70$ and $1.90 \mu \mathrm{g} / \mathrm{mL}$, respectively. It was noteworthy that acetylation of the bioactive compounds led to semisynthetic derivatives which unfortunately did not present any activity. This is the first report on the phytochemical and pharmacological investigation of Dracaena viridiflora.
\end{abstract}

Keywords: Dracaena viridiflora; steroidal saponins; epoxycholesta-5,22-dienes; cytotoxicity. (C) 2017 ACG Publications. All rights reserved.

\section{Introduction}

Dracaena viridiflora Engl \& Krause is a member of the family Agavaceae which comprises more than 480 species distributed in tropical and subtropical regions of the world [1]. Although there are no scientific reports on the phytochemical and biological studies of this plant, its leaves are used in the West Region of Cameroon for the treatment of microbial infections, epilepsy and convulsions. In our continuous search of potentially bioactive steroidal glycosides from Cameroonian medicinal plants

\footnotetext{
* Corresponding author: E-Mail: remyteponno@gmail.com (R.B. Teponno), Phone: +237677312743; opatz@uni-mainz.de (T. Opatz), Phone: +4961313922272, Fax: +4961313922338
} 
[2-5], we have investigated the $\mathrm{MeOH}$ extract from the leaves of $D$. viridiflora, leading to the isolation and structure elucidation of ten secondary metabolites including trillin $\mathbf{1}$, prosapogenin A of dioscin $\mathbf{2}$, prosapogenin B of dioscin 3, dioscin 4, gracillin 5, methylprotodioscin $\mathbf{6}$, cylicodiscoside $\mathbf{7}$, stigmasterol 8, stigmasterol 3-O- $\beta$-D-glucopyranoside 9, and allantoin 10. Since steroids have been a rich source of agents with potential pharmaceutical applications that have inspired the synthesis of new analogues with promising pharmacological activities [6], some isolated compounds were acetylated and three previously undescribed 22,26-epoxycholesta-5,22-diene derivatives 15-17 were prepared by rearrangement/acetylation in the presence of $\mathrm{ZnCl}_{2} / \mathrm{Ac}_{2} \mathrm{O}$ (Figure 1). The structures of the isolated and semisynthetic compounds were determined mainly by extensive spectroscopic analysis (1D and 2D NMR) and HRESI-MS, as well as by comparison of their spectral data with those reported in the literature. Among the compounds biologically evaluated, dioscin (4) proved to be more active against the human lung carcinoma epithelial cells A549 than doxorubicin used as positive control (Table 2).

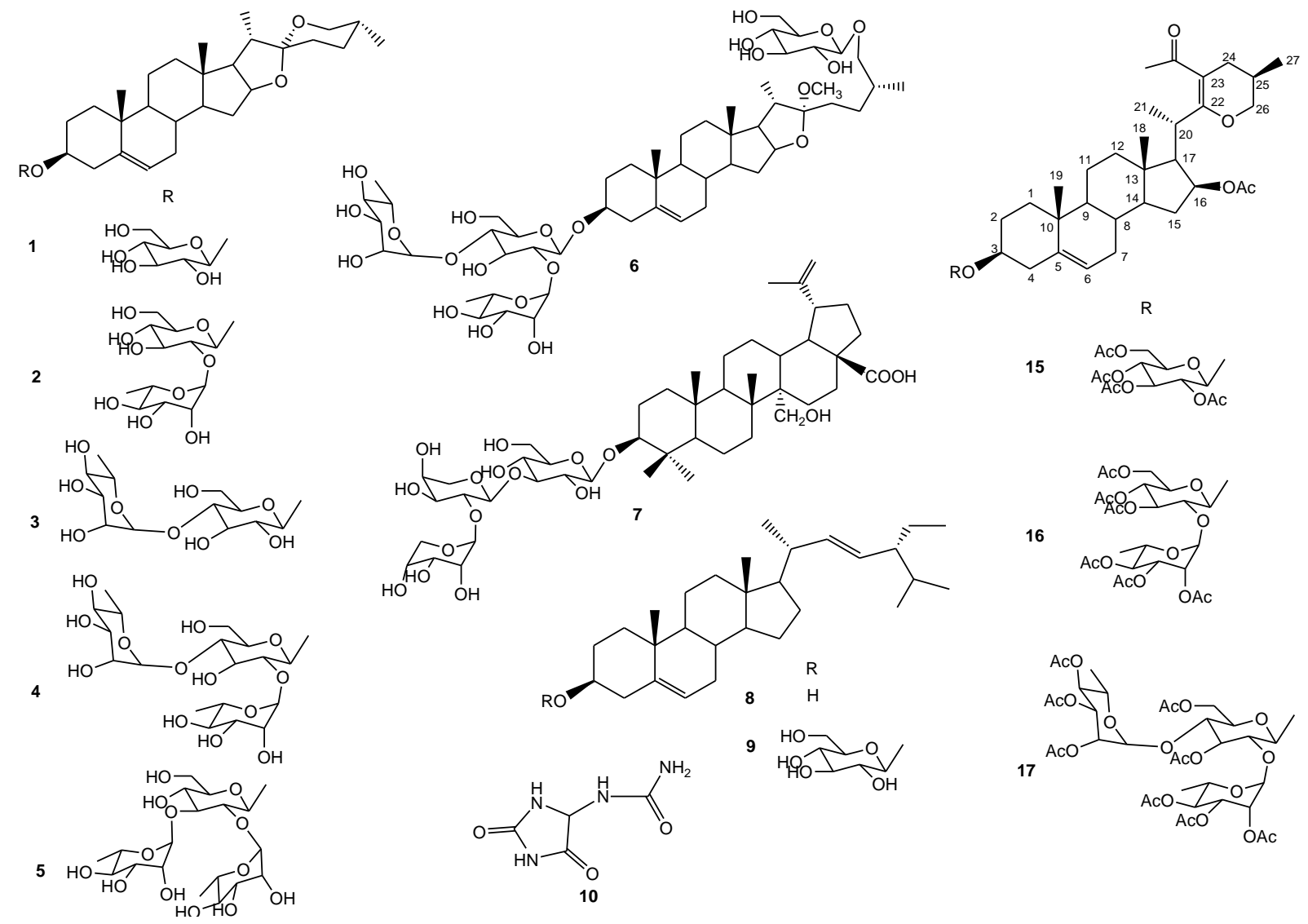

Figure 1. Structures of isolated compounds and some semisynthetic derivatives

\section{Materials and Methods}

\subsection{General Experimental Procedures}

Optical rotations were measured using the sodium D-line on a Perkin-Elmer 241 MC polarimeter at $25{ }^{\circ} \mathrm{C}$. The IR absorption spectra were measured on a Bruker Tensor 27 FT-IR using a diamond ATR. Positive ion mode HRESI mass spectra were carried out on an Agilent 6320 Ion Trap Instrument. ${ }^{1} \mathrm{H}$ and ${ }^{13} \mathrm{C}$ NMR, COSY, HSQC, HMBC and NOESY spectra were recorded in deuterated $\mathrm{MeOH}$, chloroform, pyridine and DMSO on a Bruker AVANCE III-600 MHz Spectrometer equipped 
with a $5 \mathrm{~mm}$ cryogenic TCI-probe head using standard gradient-selected pulse sequences. Column chromatography was performed using sephadex LH-20 and silica gel (Fluka analytical silica gel $90 \mathrm{C}$ 18 reversed phase for column chromatography and Macherey-Nagel Kieselgel 60 M 0.04-0.063 $\mathrm{mm} / 230-400$ mesh ASTM for column chromatography). The following solvent systems were used: $\mathrm{MeOH}$ for Sephadex column chromatography, $\mathrm{MeOH}-\mathrm{H}_{2} \mathrm{O}$ (1:1 and 6:4) for reversed phase column chromatography, Petroleum ether-Ethyl acetate 1:1 and EtOAc-MeOH- $\mathrm{H}_{2} \mathrm{O}$ (80:20:10, 85:15:10, 90:10:5, 95:5:2 and 99:1:0) for normal phase column chromatography. TLC was carried out on precoated Kieselgel $60 \mathrm{~F}_{254}$ (Merck) plates developed with EtOAc-MeOH- $\mathrm{H}_{2} \mathrm{O}$ (80:20:10, 90:10:5 and 95:5:2). Substance bands were visualised after spraying with $10 \% \mathrm{H}_{2} \mathrm{SO}_{4}$ followed by warming or directly on a $254 \mathrm{~nm}$ UV lamp for fluorescent spots.

\subsection{Plant Material}

The leaves of D. viridiflora were collected in Bangoua (West Region of Cameroon) in July 2012 and authenticated at the Cameroon National Herbarium, Yaoundé, where a voucher specimen is deposited (50698HNC).

\subsection{Extraction and isolation}

The dried and pulverized leaves of $D$. viridiflora $(1.5 \mathrm{~kg})$ were extracted with $\mathrm{MeOH}(2 \times 8 \mathrm{~L})$ for 24 hours. The filtrate obtained was concentrated under reduced pressure to yield a dark residue $(170 \mathrm{~g})$. A part of this extract $(162 \mathrm{~g})$ was submitted to silica gel $(63-200 \mu \mathrm{m})$ column chromatography $(\mathrm{CC})$, using a gradient of EtOAc in $n$-hexane, then with EtOAc-MeOH (with increasing amounts of $\mathrm{MeOH}$ ) and five main fractions A-E were obtained. Fractions A (20.8 g) (eluted with hexane to hexane-EtOAc 7:3) and B (24.3 g) (with eluted hexane-EtOAc 6:4 to hexane-EtOAc 4:6) were shown to contain mainly oily compounds and chlorophylls, respectively. Fraction C ( $3.9 \mathrm{~g})$ (eluted with hexane-EtOAc 3:7) was chromatographed using silica gel $(32-63 \mu \mathrm{m})$ with petroleum ether-EtOAc (1:1) as eluent to afford stigmasterol $8(150 \mathrm{mg})$. Fraction D (31.1 g) (EtOAc to EtOAc$\mathrm{MeOH} 8: 2)$ was purified by $\mathrm{CC}$ over silica gel $(32-63 \mu \mathrm{m})$ using EtOAc-MeOH- $\mathrm{H}_{2} \mathrm{O}(95: 5: 2)$ as eluent to yield four subfractions $\mathrm{D}_{1}-\mathrm{D}_{4}$. Subfraction $\mathrm{D}_{1}(1.5 \mathrm{~g})$ was chromatographed on a silica gel column eluted with AcOEt-MeOH (99:1) to afford trillin 1 (290 mg), allantoin $10(45 \mathrm{mg})$ and stigmasterol 3-O- $\beta$-D-glycopyranoside $9(250 \mathrm{mg})$. Subfraction $\mathrm{D}_{2}(8.5 \mathrm{~g})$ was repeatedly subjected to $\mathrm{CC}$ using EtOAc-MeOH- $\mathrm{H}_{2} \mathrm{O}(95: 5: 2)$ as eluent to yield prosapogenin A of dioscin $2(185 \mathrm{mg})$ and prosapogenin $B$ of dioscin $3(25 \mathrm{mg})$. Recrystallization of subfraction $\mathrm{D}_{3}(3.5 \mathrm{~g})$ gave a mixture $(2 \mathrm{~g})$ of dioscin and gracillin. Recristallization of subfraction $\mathrm{D}_{4}(0.5 \mathrm{~g})$ gave pure dioscin $4(75 \mathrm{mg})$. Fraction E (EtOAc-MeOH 7:3 to EtOAc-MeOH 1:1) (26.5 g) was separated on a silica gel CC using EtOAc- $\mathrm{MeOH}-\mathrm{H}_{2} \mathrm{O}(8: 2: 1)$ to yield two subsfraction $\mathrm{E}_{1}$ and $\mathrm{E}_{2}$. Subfraction E1 (10.7 g) was a complex mixture and subfraction $\mathrm{E}_{2}(1.2 \mathrm{~g})$ consisted of a mixture of very polar compounds. $\mathrm{E}_{2}$ was submitted to RP-18 CC eluted with $\mathrm{MeOH}-\mathrm{H}_{2} \mathrm{O}$ 1:1 to afford gracillin 5 (13 mg), cylicodiscoside 7 $(75 \mathrm{mg})$ and methylprotodioscin 6 (180 mg).

\subsection{Semisynthesis}

\subsubsection{Acetylation}

Trillin $(30 \mathrm{mg}, 0.052 \mathrm{mmol})$ was dissolved in pyridine $(1.5 \mathrm{~mL}), \mathrm{Ac}_{2} \mathrm{O}(1.5 \mathrm{~mL})$ was added and the mixture was stirred at room temperature overnight. Excess pyridine and $\mathrm{Ac}_{2} \mathrm{O}$ was removed by adding toluene and the mixture was concentrated to dryness under reduced pressure. The reaction 
Table $1 .{ }^{13} \mathrm{C}$ and ${ }^{1} \mathrm{H}$ NMR data (150 and $600 \mathrm{MHz}$, resp.) of compounds 15 and $\mathbf{1 6}$ in $\mathrm{CDCl}_{3}$.

\begin{tabular}{|c|c|c|c|c|}
\hline & 15 & & 16 & \\
\hline Position & $\boldsymbol{\delta}_{\mathrm{C}}$ & $\boldsymbol{\delta}_{\mathrm{H}}$ & $\boldsymbol{\delta}_{\mathrm{C}}$ & $\boldsymbol{\delta}_{\mathrm{H}}$ \\
\hline 1 & 37.3 & $1.04(\mathrm{~m}), 1.84(\mathrm{~m})$ & 37.1 & $1.08(\mathrm{~m}), 1.86(\mathrm{~m})$ \\
\hline 2 & 29.6 & $1.59(\mathrm{~m}), 1.90(\mathrm{~m})$ & 29.7 & $1.56(\mathrm{~m}), 1.96(\mathrm{~m})$ \\
\hline 3 & 80.1 & $3.48(\mathrm{~m})$ & 79.1 & $3.61(\mathrm{~m})$ \\
\hline 4 & 39.0 & $2.18(\mathrm{~m}), 2.25(\mathrm{~m})$ & 38.3 & $2.29(\mathrm{~m}), 2.43(\mathrm{~m})$ \\
\hline 5 & 140.6 & / & 140.1 & / \\
\hline 6 & 122.0 & $5.34(\mathrm{brd}, 5.1)$ & 122.1 & $5.37(\mathrm{brd}, 5.1)$ \\
\hline 7 & 31.9 & $1.94(\mathrm{o}), 2.19(\mathrm{o})$ & 31.7 & $1.96(\mathrm{o}), 2.34(\mathrm{o})$ \\
\hline 8 & 31.5 & $1.52(\mathrm{o})$ & 31.4 & $1.53(\mathrm{o})$ \\
\hline 9 & 50.3 & $0.93(\mathrm{~m})$ & 50.1 & $0.95(\mathrm{o})$ \\
\hline 10 & 36.9 & / & 36.7 & / \\
\hline 11 & 20.9 & $1.52(\mathrm{o})$ & 20.9 & $1.52(\mathrm{o})$ \\
\hline 12 & 40.0 & $1.23(\mathrm{~m}), 2.00(\mathrm{~m})$ & 39.8 & $1.25(\mathrm{~m}), 2.01(\mathrm{~m})$ \\
\hline 13 & 42.4 & I & 42.3 & / \\
\hline 14 & 54.5 & $0.97(\mathrm{~m})$ & 54.3 & $0.98(\mathrm{~m})$ \\
\hline 15 & 35.1 & $1.04(\mathrm{~m}), 2.29(\mathrm{~m})$ & 34.9 & $1.03(\mathrm{~m}), 2.30(\mathrm{~m})$ \\
\hline 16 & 75.3 & $5.13(\mathrm{~m})$ & 75.1 & $5.14(\mathrm{td}, 7.9,4.5)$ \\
\hline 17 & 56.1 & $1.81(\mathrm{o})$ & 55.9 & $1.80(\mathrm{o})$ \\
\hline 18 & 13.1 & $0.91(\mathrm{~s})$ & 13.0 & $0.91(\mathrm{~s})$ \\
\hline 19 & 19.6 & $0.99(\mathrm{~s})$ & 19.3 & $1.01(\mathrm{~s})$ \\
\hline 20 & 33.1 & $4.06(\mathrm{~m})$ & 32.9 & $4.07(\mathrm{o})$ \\
\hline 21 & 19.6 & $1.18(\mathrm{~d}, 6.9)$ & 19.5 & $1.18(\mathrm{~d}, 7.0)$ \\
\hline 22 & 171.5 & / & 171.4 & / \\
\hline 23 & 107.1 & / & 106.9 & l \\
\hline 24 & 31.8 & $2.23(\mathrm{o}), 2.44(\mathrm{o})$ & 31.7 & $2.24(\mathrm{~m}), 2.43(\mathrm{o})$ \\
\hline 25 & 26.7 & $1.89(\mathrm{~m})$ & 26.6 & $1.90(\mathrm{~m})$ \\
\hline 26 & 71.7 & $\begin{array}{l}3.45(\mathrm{t}, 10.2), 4.00(\mathrm{ddd}, 10.2, \\
2.3,1.2)\end{array}$ & 71.6 & $3.45(\mathrm{t}, 10.1), 4.00(\mathrm{~m})$ \\
\hline 27 & 17.0 & $0.97(\mathrm{~d}, 6.4)$ & 16.9 & $0.97(\mathrm{~d}, 6.4)$ \\
\hline $23-\mathrm{COCH}_{3}$ & $198.4,30.0$ & $2.20(\mathrm{~s})$ & $198.3,29.8$ & $2.20(\mathrm{~s})$ \\
\hline $16-\mathrm{OCOCH}_{3}$ & $170.9,21.4$ & $1.85(\mathrm{~s})$ & $170.7,21.2$ & $1.85(\mathrm{~s})$ \\
\hline $1^{\prime}$ & 99.8 & $4.59(\mathrm{~d}, 8.0)$ & 99.4 & $4.58(\mathrm{~d}, 7.8)$ \\
\hline $2^{\prime}$ & 71.6 & $4.96(\mathrm{dd}, 8.0,9.5)$ & 75.3 & $3.72(\mathrm{dd}, 9.5,7.8)$ \\
\hline $3^{\prime}$ & 73.0 & $5.20(\mathrm{dd}, 9.5,9.8)$ & 74.8 & $5.24(\mathrm{o})$ \\
\hline $4^{\prime}$ & 68.6 & $5.08(\mathrm{t}, 9.8)$ & 68.8 & $4.96(\mathrm{t}, 10.0)$ \\
\hline $5^{\prime}$ & 71.8 & $3.67(\mathrm{~m})$ & 71.6 & $3.68(\mathrm{ddd}, 10.0,4.9,2.5)$ \\
\hline $6^{\prime}$ & 62.2 & $\begin{array}{l}4.11(\mathrm{dd}, 12.2,2.4), 4.25(\mathrm{dd}, \\
12.2,4.8)\end{array}$ & 62.2 & $\begin{array}{l}4.07 \text { (o), } 4.27 \text { (dd, 12.2, } \\
4.9)\end{array}$ \\
\hline 2'-OCOCH & $169.5,20.9$ & $2.05(\mathrm{~s})$ & l & l \\
\hline $3^{\prime}-\mathrm{OCOCH}_{3}$ & $170.6,20.8$ & $2.00(\mathrm{~s})$ & $170.3,20.8$ & $2.06(\mathrm{~s})$ \\
\hline $4^{\prime}-\mathrm{OCOCH}_{3}$ & $169.6,20.9$ & $2.02(\mathrm{~s})$ & $169.7,20.9$ & $1.99(\mathrm{~s})$ \\
\hline 6'-OCOCH 3 & $170.9,21.0$ & $2.08(\mathrm{~s})$ & $170.7,21.0$ & $2.07(\mathrm{~s})$ \\
\hline $1 "$ & & & 97.3 & $4.96(\mathrm{~d}, 2.0)$ \\
\hline $2^{\prime \prime}$ & & & 70.2 & $4.99(\mathrm{dd}, 3.4,1.8)$ \\
\hline $3 "$ & & & 68.6 & $5.23(\mathrm{o})$ \\
\hline $4 "$ & & & 71.2 & $5.07(\mathrm{t}, 10.0)$ \\
\hline $5^{\prime \prime}$ & & & 66.4 & $4.39(\mathrm{~m})$ \\
\hline $6 "$ & & & 17.3 & $1.19(\mathrm{~d}, 6.3)$ \\
\hline $2 "-\mathrm{OCOCH}_{3}$ & & & $169.9,20.8$ & $2.00(\mathrm{~s})$ \\
\hline $3 "-\mathrm{OCOCH}_{3}$ & & & $170.0,20.7$ & $2.02(\mathrm{~s})$ \\
\hline 4"-- $\mathrm{OCOCH}_{3}$ & & & $170.1,21.0$ & $2.12(\mathrm{~s})$ \\
\hline
\end{tabular}

o: overlapped signals

mixture was purified by chromatography on a silica gel column eluted with petroleum ether-EtOAc (7:3) to give trillin tetraacetate ( $35 \mathrm{mg}, 93 \%)$. Prosapogenin A of dioscin hexaacetate (38 mg, 94\%) 
was prepared from $30 \mathrm{mg}$ of prosapogenin A of dioscin following the same procedure. Furthermore, a mixture of dioscin and gracillin $(1.1 \mathrm{~g})$ was acetylated to yield after purification dioscin octaacetate (400 mg), gracillin octaacetate $(282 \mathrm{mg}$ ) and a mixture of both compounds $(420 \mathrm{mg})$.

\subsubsection{Reaction with $\mathrm{ZnCl}_{2} / \mathrm{Ac}_{2} \mathrm{O}$}

To a suspension of trillin tetraacetate $(10 \mathrm{mg}, 0.0134 \mathrm{mmol})$ in $2 \mathrm{~mL}$ of $\mathrm{Ac}_{2} \mathrm{O}$ was added $2 \mathrm{mg}$ $(0.0147 \mathrm{mmol})$ of anhydrous $\mathrm{ZnCl}_{2}$. The reaction mixture was stirred for $20 \mathrm{~h}$ at room temperature and quenched with ice. The organic phase was extracted with ethyl acetate, neutralized with a saturated $\mathrm{NaHCO}_{3}$ solution, dried over anhydrous $\mathrm{MgSO}_{4}$, and evaporated under reduced pressure to yield a powder which was chromatographed on a silica gel column eluted with petroleum ether-EtOAc $(7: 4)$ to give $8 \mathrm{mg}$ of compound $\mathbf{1 5}(72 \%)$. Prosapogenin A of dioscin hexaacetate $(20 \mathrm{mg}, 0.020 \mathrm{mmol})$ and dioscin octaacetate $(30 \mathrm{mg}, 0.025 \mathrm{mmol}$ ) were submitted in the same reaction conditions to give $15 \mathrm{mg}$ of compound (16) (69\%) and $26 \mathrm{mg}$ of compound $\mathbf{1 7}(81 \%)$, respectively.

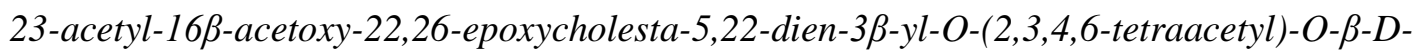
glucopyranoside (15): White amorphous powder; $[\alpha]_{\mathrm{D}}{ }^{25}-8.5\left(c=0.17, \mathrm{CHCl}_{3}\right)$; $\mathrm{IR} v_{\max }$ (ATR): 2939, 1757, 1664, 1568, $1222 \mathrm{~cm}^{-1} ;{ }^{13} \mathrm{C}$ and ${ }^{1} \mathrm{H}$ NMR data: see Table 1; HRESI-MS: $m / z 829.4368[\mathrm{M}+\mathrm{H}]^{+}$ (Calcd for $\mathrm{C}_{45} \mathrm{H}_{65} \mathrm{O}_{14}: 829.4369$ ).

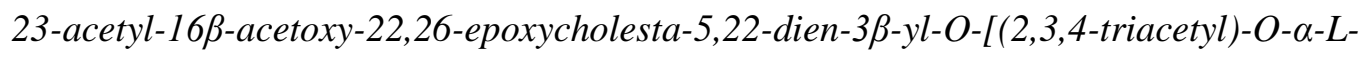
rhamnopyranosyl-( $(1 \rightarrow 2)$-O-(3,4,6-triacetyl)]-O- $\beta$-D-glucopyranoside $(\mathbf{1 6})$ : White amorphous powder; $[\alpha]_{\mathrm{D}}{ }^{25}-10.2\left(c=1.11, \mathrm{CHCl}_{3}\right) ; \mathrm{IR} v_{\max }$ (ATR): $2939,1749,1667,1575,1224 \mathrm{~cm}^{-1} ;{ }^{13} \mathrm{C}$ and ${ }^{1} \mathrm{H}$ NMR data: see Table 1; HRESI-MS: $m / z 1081.4969[\mathrm{M}+\mathrm{Na}]^{+}\left(\mathrm{Calcd}\right.$ for $\mathrm{C}_{55} \mathrm{H}_{78} \mathrm{O}_{20} \mathrm{Na}$ : 1081.4979).

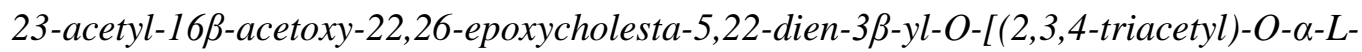
rhamnopyranosyl-( $(\rightarrow 2)$-O-(2,3,4-triacetyl $)-O$ - $\alpha$-L-rhamnopyranosyl-( $(\rightarrow 4)]-O-(3,6$-diacetyl $)-O-\beta$ D-glucopyranoside (17): White amorphous powder; $[\alpha]_{\mathrm{D}}{ }^{25}-24.3\left(c=1.00, \mathrm{CHCl}_{3}\right) . \mathrm{IR} v_{\max }$ (ATR): 2936, 1750, 1667, 1575, $1221 \mathrm{~cm}^{-1} ;{ }^{13} \mathrm{C}$ and ${ }^{1} \mathrm{H}$ NMR data: see Table 2; HRESI-MS: $\mathrm{m} / \mathrm{z} 1289.5986$ $[\mathrm{M}+\mathrm{Na}]^{+}\left(\right.$Calcd for $\left.\mathrm{C}_{65} \mathrm{H}_{93} \mathrm{O}_{26} \mathrm{Na}: 1289.5950\right)$.

\subsection{Cytotoxic activity}

\subsubsection{Cell lines and culture conditions}

The effect of compounds on cell growth was evaluated against human cancer cells including lung adenocarcinoma A549, human epithelial colorectal adenocarcinoma cells Caco-2, human ovarian carcinoma Skov-3 and human T-Cell leukemia cells JURKAT. SKOV-3 was cultured in IMDM (Iscove's Modified Dulbecco's Medium), Caco-2 and A549 were cultured on DMEM (Dulbecco's Modified Eagle Medium) high glucose with sodium pyruvate while JURKAT was maintained on RPMI medium containing $\beta$-mercaptoethanol $0.05 \mathrm{mM}$. All media were supplemented with $10 \%$ fetal bovine serum (FBS) and $1 \%$ penicillin/streptomycin/fungizone (PSF) solution. The cell lines were cultured at $37^{\circ} \mathrm{C}$ in a humidified environment containing $5 \% \mathrm{CO}_{2}$.

\subsubsection{MTT assay}

In vitro cytotoxicity test against above mentioned cell lines was performed by the [3-(4,5Dimethylthiazol-2-yl)-2,5-diphenyltetrazolium bromide] MTT assay. Briefly, cells were harvested in $\log$ phase using trypsin $(0.05 \%$ trypsin, $0.02 \%$ EDTA, in PBS). The cell suspensions were diluted with appropriate growth medium to obtain the cell density of $10^{4}$ cells/well. Aliquots of $100 \mu \mathrm{L}$ of each suspension were seeded in 96 wells cell culture plates. The cells were incubated at $37^{\circ} \mathrm{C}$ in an 
atmosphere of $5 \% \mathrm{CO}_{2}$ and $95 \%$ relative humidity in a $\mathrm{CO}_{2}$ incubator. After $24 \mathrm{~h}$ of incubation, test materials $(100 \mu \mathrm{L} /$ well $)$ at varying concentrations were added to the wells containing cells. Doxorubicin was used as positive control. Suitable controls with equivalent concentration of DMSO were also included. The plates were further incubated for $48 \mathrm{~h}$, then the medium in each well was aspirated and MTT solution ( $2 \mathrm{mg} / \mathrm{mL}$ in PBS) was diluted 1:10 with fresh medium and added to each well and the plates were incubated at $37^{\circ} \mathrm{C}$ for $4 \mathrm{~h}$. The medium was aspirated from the wells and DMSO was added to solubilize the formed formazan crystals. The absorbance was measured on FLUOstar OPTIMA microplate reader at $570 \mathrm{~nm}$. The concentration causing 50\% inhibition of cell growth $\left(\mathrm{IC}_{50}\right)$ was calculated from the concentration-inhibition response curve by regression analysis.

\section{Results and Discussion}

\subsection{Structure elucidation}

Phytochemical investigation of the $\mathrm{MeOH}$ extract from the leaves of Dracaena viridiflora, led to the isolation of trillin 1 [7], prosapogenin A of dioscin 2 [8], prosapogenin B of dioscin 3 [9], dioscin 4 [8], gracillin 5 [10], methylprotodioscin 6 [10], cylicodiscoside 7 [11]. Stigmasterol 8 [12], stigmasterol 3-O-glucopyranoside 9 [12], and allantoin 10 [13]. Furthermore, trillin tetraacetate 11 [14], prosapogenin A of dioscin hexaacetate 12 [15], dioscin octaacetate 13 [14], gracillin octaacetate 14 [14] were prepared and three previously undescribed 22,26-epoxycholesta-5,22-diene derivatives 15-17 were synthesized by rearrangement/acetylation of trillin tetraacetate, prosapogenin A of dioscin hexaacetate and dioscin octaacetate, respectively in the presence of $\mathrm{ZnCl}_{2} / \mathrm{Ac}_{2} \mathrm{O}$.

Compound 15 was obtained as a white amorphous powder from petroleum ether-EtOAc (7:4). Its molecular formula $\mathrm{C}_{45} \mathrm{H}_{64} \mathrm{O}_{14}$ was determined from the HRESI-MS which showed the pseudomolecular ion peak at $\mathrm{m} / z 829.4368[\mathrm{M}+\mathrm{H}]^{+}$. The presence of acetyl and conjugated carbonyl groups was deduced from the IR absorption bands at 1757 and $1664 \mathrm{~cm}^{-1}$, respectively. The ${ }^{1} \mathrm{H}$ NMR spectrum of 15 showed signals for four steroid methyl groups at $\delta 1.18$ (d, $J=6.9 \mathrm{~Hz}, \mathrm{H}-21), 0.99$ (s, H-19), 0.97 (d, $J=6.4 \mathrm{~Hz}, \mathrm{H}-27$ ) and 0.91 (s, H-18), an acetyl methyl protons singlet at $\delta 2.20$ (s, Ac23) as well as an olefinic proton at $\delta 5.34$ (brd, $J=5.1 \mathrm{~Hz}, \mathrm{H}-6$ ). It also exhibited signals for two oxymethyne protons at $\delta 3.48(\mathrm{~m}, \mathrm{H}-3)$ and $5.13(\mathrm{~m}, \mathrm{H}-16)$, the methylene protons at $\delta 4.00$ (ddd, $J=$ $10.2,2.3,1.2, \mathrm{H}-26 \mathrm{a})$ and $3.45(\mathrm{t}, J=10.2, \mathrm{H}-26 \mathrm{~b})$. The above proton signals together with distinctive carbon signals at $\delta 198.4$ (Ac-23), 171.5 (C-22), 140.6 (C-5), 122.0 (C-6), 107.1 (C-23), 80.1 (C-3), 75.3 (C-16) and 71.7 (C-26) indicated that the aglycone part was of the 22,26-epoxycholesta-5,22diene type $[6,16]$. The sugar part of the ${ }^{1} \mathrm{H}$ NMR spectrum showed the anomeric proton signal at $\delta$ $4.59\left(\mathrm{~d}, J=8.0 \mathrm{~Hz}, \mathrm{H}-1^{\prime}\right)$ which gave HSQC correlation with carbon at $\delta 99.8\left(\mathrm{C}-1^{\prime}\right)$ and some deshielded proton signals at $\delta 4.96\left(\mathrm{dd}, J=8.0,9.5 \mathrm{~Hz}, \mathrm{H}-2^{\prime}\right), 5.20\left(\mathrm{dd}, J=9.5,9.8 \mathrm{~Hz}, \mathrm{H}-3^{\prime}\right)$ and 5.08 (t, $J=9.8 \mathrm{HZ}, \mathrm{H}-4^{\prime}$ ) due to acetylation shifts [17]. In the ${ }^{1} \mathrm{H}$ NMR spectrum of compound 15, the presence of acetoxy groups was indicated by methyl singlets at $\delta 2.08$ (OAc-6'), 2.05 (OAc-2'), 2.02 $\left(\mathrm{OAc}-4^{\prime}\right), 2.00\left(\mathrm{OAc}^{\prime} 3^{\prime}\right), 2.08\left(\mathrm{OAc}-6^{\prime}\right), 1.85$ (OAc-16), whereas in the ${ }^{13} \mathrm{C}$ NMR spectrum, the signals of ester carbonyls at $\delta 170.9$ (OAc-16 and OAc-6'), 170.6 (OAc-3'), 169.6 (OAc-4'), and 169.5 $\left(\mathrm{OAc}-2^{\prime}\right)$ were observed. Intensive interpretation of the ${ }^{1} \mathrm{H}$ and ${ }^{13} \mathrm{C} \mathrm{NMR},{ }^{1} \mathrm{H}-{ }^{1} \mathrm{H}$ COSY, HSQC and HMBC spectra (Table 1) and comparison with published data led to the elucidation of $\mathbf{1 5}$ as 23-acetyl$16 \beta$-acetoxy-22,26-epoxycholesta-5,22-dien-3 $\beta$-yl- $O$-(2,3,4,6-tetraacetyl)- $O$ - $\beta$-D-glucopyranoside .

Compounds 16 and 17 were also isolated as white amorphous powders. The ${ }^{1} \mathrm{H}$ and ${ }^{13} \mathrm{C}$ NMR data of their aglycone parts (Tables 1 and 2) were very similar to those of $\mathbf{1 5}$, indicating that they derived from the same rearrangement. Their HRESI-MS spectra exhibited pseudomolecular ion peaks at $\mathrm{m} / z .1081 .4969$ and $1289.5986[\mathrm{M}+\mathrm{Na}]^{+}$corresponding to the molecular formulas $\mathrm{C}_{55} \mathrm{H}_{78} \mathrm{O}_{20}$ and $\mathrm{C}_{65} \mathrm{H}_{93} \mathrm{O}_{26}$, respectively. Their structures were established as 23-acetyl-16 $\beta$-acetoxy-22,26-

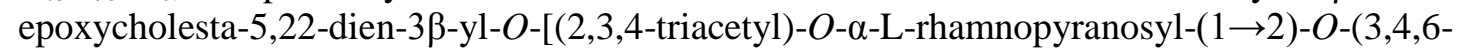

triacetyl)]-O- $\beta$-D-glucopyranoside (16) and 23-acetyl-16 $\beta$-acetoxy-22,26-epoxycholesta-5,22-dien-3 $\beta$ yl-O-[(2,3,4-triacetyl)- $O$ - $\alpha$-L-rhamnopyranosyl-( $1 \rightarrow 2)-O$-(2,3,4-triacetyl)- $O$ - $\alpha$-L-rhamnopyranosyl- 
$(1 \rightarrow 4)]-O$-(3,6-diacetyl)- $O$ - $\beta$-D-glucopyranoside $(17)$ from careful examination of the ${ }^{1} \mathrm{H}$ and ${ }^{13} \mathrm{C}$ NMR, HSQC, HMBC and ${ }^{1} \mathrm{H}_{-}{ }^{1} \mathrm{H}$ COSY spectra. The plausible mechanism of the rearrangement/acetylation of spirostane steroids in the presence of $\mathrm{ZnCl}_{2} / \mathrm{Ac}_{2} \mathrm{O}$ (Scheme 1) was proposed Sandoval-Ramírez et al. [18].

Table 2. ${ }^{13} \mathrm{C}$ and ${ }^{1} \mathrm{H}$ NMR data (150 and $600 \mathrm{MHz}$, resp.) of compound $17 \mathrm{in} \mathrm{CDCl}_{3}$.

\begin{tabular}{|c|c|c|c|c|c|}
\hline Position & $\boldsymbol{\delta}_{\mathrm{C}}$ & $\delta_{\mathrm{H}}$ & Position & $\boldsymbol{\delta}_{\mathrm{C}}$ & $\delta_{\mathrm{H}}$ \\
\hline 1 & 37.3 & $1.09(\mathrm{~m}), 1.85(\mathrm{~m})$ & $1^{\prime}$ & 99.7 & $4.56(\mathrm{~d}, 7.8)$ \\
\hline 2 & 29.7 & $1.56(\mathrm{~m}), 1.96(\mathrm{~m})$ & $2^{\prime}$ & 76.4 & $3.57(t, 7.8)$ \\
\hline 3 & 79.2 & $3.60(\mathrm{o})$ & $3^{\prime}$ & 75.5 & $5.28(t, 9.4)$ \\
\hline 4 & 38.4 & $2.27(\mathrm{~m}), 2.43(\mathrm{~m})$ & $4^{\prime}$ & 77.8 & $3.73(t, 9.5)$ \\
\hline 5 & 140.2 & l & $5^{\prime}$ & 72.4 & $3.60(\mathrm{o})$ \\
\hline 6 & 122.2 & $5.36(\mathrm{brd}, 5.0)$ & 6 & 62.4 & $\begin{array}{l}4.29(\mathrm{dd}, 12.3,3.8) \\
4.44(\mathrm{dd}, 12.3,1.5)\end{array}$ \\
\hline 7 & 31.8 & $1.95(\mathrm{o}), 2.26(\mathrm{o})$ & 2'- $-\mathrm{OCOCH}_{3}$ & I & I \\
\hline 8 & 31.5 & $1.52(\mathrm{o})$ & $3^{\prime}-\mathrm{OCOCH}_{3}$ & $170.4,21.1$ & $2.14(\mathrm{~s})$ \\
\hline 9 & 50.2 & $0.96(\mathrm{o})$ & 4'- $-\mathrm{OCOCH}_{3}$ & l & I \\
\hline 10 & 36.9 & l & 6'--OCOCH 3 & $170.9,21.0$ & $2.09(\mathrm{~s})$ \\
\hline 11 & 21.0 & $1.52(\mathrm{o})$ & $1 "$ & 97.4 & $4.90(\mathrm{~d}, 1.7)$ \\
\hline 12 & 39.9 & $1.25(\mathrm{o}), 1.99(\mathrm{o})$ & $2 "$ & 70.4 & $5.00(\mathrm{o})$ \\
\hline 13 & 42.4 & I & 3" & 68.7 & $5.23(\mathrm{dd}, 10.0,3.4)$ \\
\hline 14 & 54.5 & $0.98(\mathrm{~m})$ & 4" & 71.4 & $5.05(\mathrm{o})$ \\
\hline 15 & 35.1 & $1.04(\mathrm{~m}), 2.29(\mathrm{~m})$ & $5 "$ & 66.6 & $4.37(\mathrm{~m})$ \\
\hline 16 & 75.3 & $5.14(\mathrm{~m})$ & 6" & 17.5 & $1.16(\mathrm{~d}, 6.1)$ \\
\hline 17 & 56.1 & $1.80(\mathrm{o})$ & $2 "-\mathrm{OCOCH}_{3}$ & $170.2,21.0$ & $2.01(\mathrm{~s})$ \\
\hline 18 & 13.1 & $0.90(\mathrm{~s})$ & 3"-OCOCH & $169.9,20.9$ & $1.98(\mathrm{~s})$ \\
\hline 19 & 19.4 & $1.00(\mathrm{~s})$ & 4"--OCOCH & $170.2,21.1$ & $2.13(\mathrm{~s})$ \\
\hline 20 & 33.1 & $4.07(\mathrm{~m})$ & 1"' & 99.7 & $4.79(\mathrm{~d}, 1.9)$ \\
\hline 21 & 19.6 & $1.17(\mathrm{~d}, 7.0)$ & $2 " \prime$ & 70.2 & $5.01(\mathrm{o})$ \\
\hline 22 & 171.6 & l & 3"' & 68.7 & $5.16(\mathrm{dd}, 10.0,3.5)$ \\
\hline 23 & 107.1 & l & 4"' & 70.7 & $5.00(\mathrm{o})$ \\
\hline 24 & 31.9 & $2.24(\mathrm{~m}), 2.42(\mathrm{o})$ & $5 " '$ & 68.1 & $3.84(\mathrm{~m})$ \\
\hline 25 & 26.7 & $1.90(\mathrm{~m})$ & 6"' & 17.3 & $1.15(\mathrm{~d}, 6.2)$ \\
\hline 26 & 71.7 & $3.45(\mathrm{t}, 10.1), 4.00(\mathrm{~m})$ & $2 " '-\mathrm{OCOCH}_{3}$ & $170.2,21.0$ & $2.04(\mathrm{~s})$ \\
\hline 27 & 17.1 & $0.96(\mathrm{~d}, 6.4)$ & 3"'--OCOCH 3 & $170.3,20.9$ & $1.98(\mathrm{~s})$ \\
\hline $23-\mathrm{COCH}_{3}$ & $198.4,29.8$ & $2.19(\mathrm{~s})$ & $4 " '-\mathrm{OCOCH}_{3}$ & $170.2,21.1$ & $2.13(\mathrm{~s})$ \\
\hline $16-\mathrm{OCOCH}_{3}$ & $170.9,21.5$ & $1.84(\mathrm{~s})$ & & & \\
\hline
\end{tabular}

\subsection{Cytotoxicity}

The isolated compounds and the crude $\mathrm{MeOH}$ extract were screened for their cytotoxic effect against Jurkat, $\mathrm{CaCo} 2, \mathrm{Skov}-3$, and A549 cancer cell lines using MTT assay. Their median inhibitory concentrations $\left(\mathrm{IC}_{50}\right)$ are listed in Table 3 . The crude extract showed significant activity with $\mathrm{IC}_{50}$ values ranged between $11.76 \mu \mathrm{g} / \mathrm{mL}$ and $23.69 \mu \mathrm{g} / \mathrm{mL}$. Out of the eleven compounds tested, three secondary metabolites (dioscin 4, prosapogenin A of dioscin 2 and methylprotodioscin 8 ) showed strong to moderate cytotoxic activity ( $\mathrm{IC}_{50}$ values varying from $0.42 \mu \mathrm{g} / \mathrm{mL}$ and $16.13 \mu \mathrm{g} / \mathrm{mL}$ ) while three others compounds (trillin 1, trillin tetraacetate $\mathbf{1 1}$ and prosapogenin B of dioscin $\mathbf{3}$ ) exhibited moderate to weak activity (with $\mathrm{IC}_{50}$ values ranged from $13.72 \mu \mathrm{g} / \mathrm{mL}$ and $93.46 \mu \mathrm{g} / \mathrm{mL}$ ) against all the four cell lines used. Dioscin showed the most potent cytotoxicity against A549, Jurkat and Skov-3 with respective $\mathrm{IC}_{50}$ values of $0.42,1.70$ and $1.90 \mu \mathrm{g} / \mathrm{mL}$. 

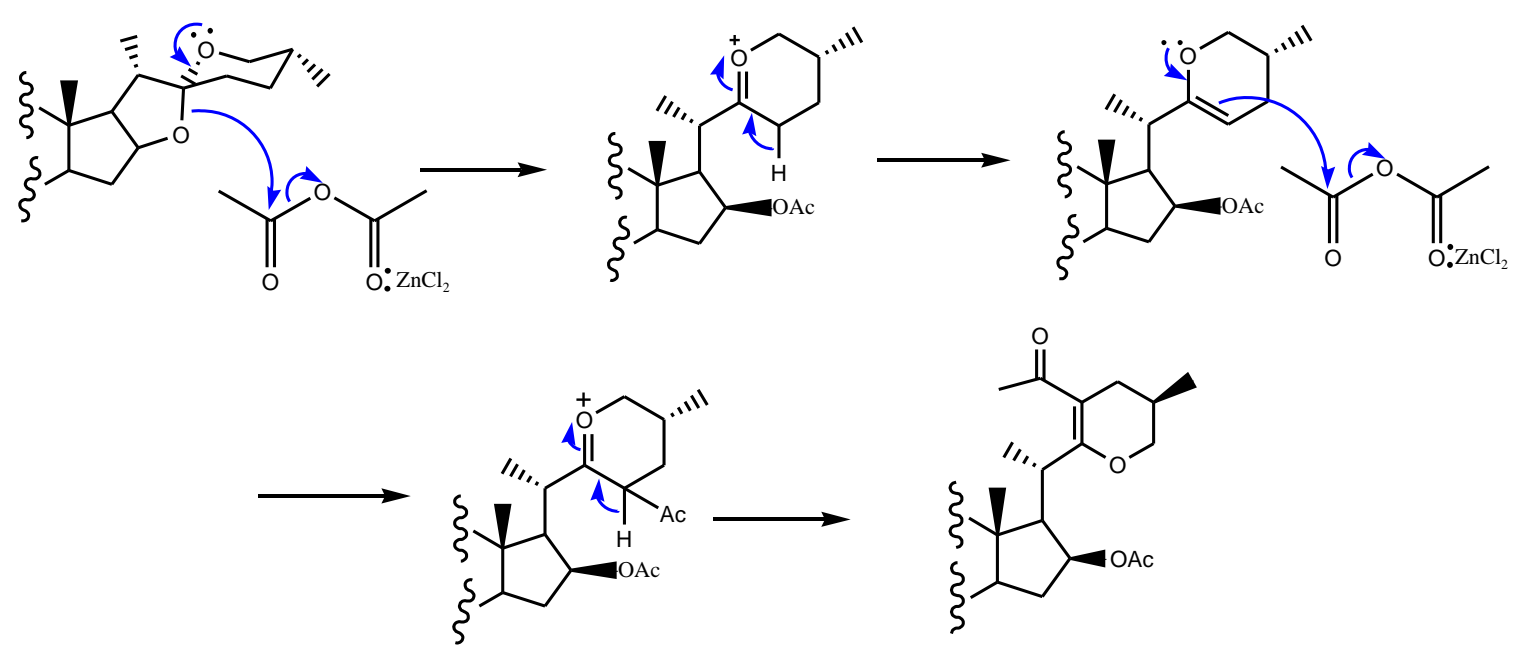

Scheme 1. Plausible mechanism for the formation of compounds 15-17 [18].

Regarding the structure-activity relationships of the investigated compounds, some features that might influence the cytotoxic activity can be drawn when comparing their chemical structures. The most active compound, dioscin 4, contains a 2,4-di- $O$-rhamnopyranosyl glucopyranoside unit linked to $\mathrm{C}$-3. In comparison with prosapogenin A of dioscin 2 (containing a 2-O-rhamnopyranosyl glucopyranoside unit) and prosapogenin $\mathrm{B}$ of dioscin $\mathbf{3}$ (containing a 4-O-rhamnopyranosyl glucopyranoside unit), the 2,4-disubstitution pattern of the glucopyranosyl unit linked to C-3 turned to be essential for the cytotoxicity. Morever, the absence of a rhamnopyranosyl unit at C-4 of the glucopyranosyl unit linked to C-3 of the aglycone considerably reduces the activity. Besides, this activity is reduced when the spirostane skeleton turns to furostane with a glucopyranosyl unit at C-26 when comparing 4 and methylprotodioscin $\mathbf{6}$. This observation is in agreement with several previous studies which reported that the biological activity of saponins is influenced both by the aglycone and the sugar moiety [19]. Except for trillin tetraacetate 11, all the acetylated compounds were not active, even the new 22,26-epoxycholesta-5,22-diene derivatives.

\section{Conclusion}

Phytochemical investigation of the $\mathrm{MeOH}$ extract from the leaves of $D$. viridiflora led to the isolation and structure elucidation of ten secondary metabolites 1-10. Furthermore, three previously unreported 22,26-epoxycholesta-5,22-diene derivatives were prepared by $\mathrm{ZnCl}_{2}$ catalysed rearrangement/acetylation reaction. Although the semisynthetic derivatives showed not activity, the significant cytotoxicity exhibited by the methanol extract of this medicinal plant could be attributed to the presence of dioscin which proved to be more active against the human lung carcinoma epithelial cells A549 than doxorubicin used as positive control. To the best of our knowledge, this is the first report on the phytochemical and pharmacological investigation of $D$. viridiflora as well as the preparation of 22,26-epoxycholesta-5,22-diene derivatives from trillin, prosapogenin A of dioscin and dioscin. In addition, cylicodiscoside is reported here for the first time from a plant of the Agavaceae family. 
Table 3. Cytotoxicity of extract, compounds and semisynthetic derivatives from $D$. viridiflora against human cancer cells lines.

\begin{tabular}{lllll}
\hline Compounds & \multicolumn{4}{c}{$\mathbf{I C}_{\mathbf{5 0}}(\boldsymbol{\mu g} / \mathbf{m L})$} \\
\cline { 2 - 5 } & Jurkat & CaCo2 & $\mathbf{S k o v - 3}$ & A549 \\
\hline MeOH Extract & $11.76 \pm 0.88^{\mathrm{a}}$ & $19.88 \pm 0.44^{\mathrm{a}}$ & $16.08 \pm 1.13^{\mathrm{a}}$ & $23.69 \pm 0.57^{\mathrm{a}}$ \\
$\mathbf{1}$ & $22.36 \pm 1.40^{\mathrm{c}}$ & $36.49 \pm 2.14^{\mathrm{c}}$ & $64.78 \pm 1.91^{\mathrm{c}}$ & $14.14 \pm 0.10^{\mathrm{c}}$ \\
$\mathbf{2}$ & $2.06 \pm 0.12^{\mathrm{b}}$ & $2.51 \pm 0.32^{\mathrm{b}}$ & $5.69 \pm 0.88^{\mathrm{e}}$ & $2.11 \pm 0.54^{\mathrm{e}}$ \\
$\mathbf{3}$ & $21.74 \pm 1.80^{\mathrm{c}}$ & $13.72 \pm 0.84^{\mathrm{e}}$ & $62.33 \pm 1.42^{\mathrm{c}}$ & $42.44 \pm 1.60^{\mathrm{f}}$ \\
$\mathbf{4}$ & $1.70 \pm 0.38^{\mathrm{b}}$ & $2.58 \pm 0.21^{\mathrm{b}}$ & $1.90 \pm 0.86^{\mathrm{b}}$ & $0.42 \pm 0.15^{\mathrm{b}}$ \\
$\mathbf{6}$ & $4.82 \pm 0.33^{\mathrm{e}}$ & $16.13 \pm 0.34^{\mathrm{f}}$ & $7.07 \pm 0.39^{\mathrm{f}}$ & $5.26 \pm 0.29^{\mathrm{g}}$ \\
$\mathbf{7}$ & $>100$ & $>100$ & $>100$ & $>100$ \\
$\mathbf{1 1}$ & $20.32 \pm 1.18^{\mathrm{c}, \mathrm{d}}$ & $42.75 \pm 5.52^{\mathrm{d}}$ & $93.46 \pm 3.59^{\mathrm{d}}$ & $18.48 \pm 0.71^{\mathrm{d}}$ \\
$\mathbf{1 2}$ & $>100$ & $>100$ & $>100$ & $>100$ \\
$\mathbf{1 3}$ & $>100$ & $>100$ & $>100$ & $>100$ \\
$\mathbf{1 4}$ & $>100$ & $>100$ & $>100$ & $>100$ \\
$\mathbf{1 7}$ & $>100$ & $>100$ & $>100$ & $>100$ \\
Doxorubicin & $0.61 \pm 0.04^{\mathrm{f}}$ & $2.32 \pm 1.04^{\mathrm{b}}$ & $0.84 \pm 0.08^{\mathrm{g}}$ & $1.15 \pm 0.84^{\mathrm{b}}$
\end{tabular}

Data represent the mean \pm SD of three independent experiments; values with different letters are significantly different at $\mathrm{p}<$ 0.05 , according to the Fisher's least significant difference (LSD) analysis. In bold are values of significant activity

\section{Acknowledgments}

This work was supported by the Rhineland-Palatinate Natural Products Research Center as well as by TWAS-DFG through the granting of a cooperation visit at the Institute of Organic Chemistry, Johannes Gutenberg University Mainz, Germany to R.B. Teponno. J.P. Dzoyem is grateful to the Philipps-Universität Marburg for a 3 months grant and to the German Academic Exchange Service (DAAD) for another 3 months research stay grant at Philipps-Universität Marburg. We thank Dr. Johannes C. Liermann as well as Dr. N. Hanold (deceased) of Johannes Gutenberg University for recording NMR and mass spectra.

\section{Supporting Information}

Supporting Information accompanies this paper on http://www.acgpubs.org/RNP

\section{References}

[1] A. Yokosuka, Y. Mimaki and Y. Sashida (2000). Steroidal saponins froms Dracaena surculosa, J. Nat. Prod. 62, 1239-1243.

[2] L. A. Tapondjou, B. K. Ponou, R. B. Teponno, M. Mbiantcha, J. D. Djoukeng, T. B. Nguelefack, P. Watcho, A. Gomez-Cadenas and H.-J. Park (2008). In Vivo anti-Inflammatory effect of a new steroidal saponin, mannioside A, and its derivatives isolated from Dracaena mannii, Arch. Pharm. Res. 31, 653658.

[3] R.B. Teponno, B. K. Ponou, D. Fiorini, L. A. Tapondjou and L. Barboni (2013). Chemical constituents from the roots of Furcraea bedinghausii Koch, Int. Lett. Chem., Phys. Astron. 11, 9-19. 
[4] R. T. Fouedjou, R. B. Teponno, L. Quassinti, M. Bramucci, D. Petrelli, L. A. Vitali, D. Fiorini, L. A. Tapondjou and L. Barboni (2014). Steroidal saponins from Cordyline fructicosa (L.) A. Chev. and their cytotoxic and antimicrobial activity, Phytochem. Lett. 7, 62-68.

[5] R. B. Teponno, C. Tanaka, B. Jie, L. A. Tapondjou and T. Miyamoto (2016). Trifasciatosides A-J, steroidal saponins from Sansevieria trifasciata, Chem. Pharm. Bull. 64, 1347-1355.

[6] J. O. H. Perez-Diaz, J. L. Vega-Baez, J. Sandoval-Ramirez, S. Meza-Reyes, S. Montiel-Smith, N. Farfan and R. Santillan (2010). Novel steroidal penta- and hexacyclic compounds derived from 12oxospirostan sapogenins, Steroids 75, 1127-1136.

[7] G. X. Wang, D. X. Jiang, J. Li, J. Han, Y.T. Liu, X. L. Liu (2010). Anthelmintic activity of steroidal saponins from Dioscorea zingiberensis $\mathrm{CH}$ Wright against Dactylogyrus intermedius (Monogenea) in goldfish (Carassius auratus), Parasitol. Res. 107, 1365-1371.

[8] K. Hu, A. J. Dong, X. S. Yao, H. Kobayashi and S. Iwasaki (1997). A furostanol glycoside from the rhizomes of Dioscorea collettii var hypoglauca, Phytochemistry 44, 1339-1342.

[9] K. Inoue, S. Kobayashi, H. Noguchi, U. Sankawa and Y. Ebizuka (1995). Spirostanol and furostanol glycosides of Costus speciosus (Koenig), Nat. Med. 49, 336-339.

[10] D.-J. Yang, T.-J. Lu and L. S. Hwang (2003). Isolation and identification of steroidal saponins in Taiwanese yam cultivar (Dioscorea pseudojaponica Yamamoto), J. Agric. Food Chem. 51, 6438-6444.

[11] M. Tene, P. Chabert, O. Note, T. J. N. Kenla, P. Tane and A. Lobstein (2011). Triterpenoid saponins from Cylicodiscus gabunensis, Phytochem. Lett. 4, 89-92.

[12] Y. C. Koay, K. C. Wong, H. Osman, I. Eldeen and M. Z. Asmawi (2013). Chemical constituents and biological activities of Strobilanthes crispus L, Rec. Nat. Prod. 7, 59-64.

[13] K. H. Kim, M. A. Kim, E. Moon, S. Y. Kim, S. Z. Choi, M. W. Son and K. R. Lee (2011). Furostanol saponins from the rhizomes of Dioscorea japonica and their effects on NGF induction, Bioorg. Med. Chem. Lett, 21, 2075-2078.

[14] U. Prawat, P. Tuntiwachwuttikul and W.C. Taylor (1989). Steroidal saponins of Costus lacerus, J. Sci. Soc. Thailand 15, 139-147.

[15] M. Miyamura, K. Nakano, T. Nohara, T. Tomimatsu and T. Kawasaki (1982). Steroid saponins from Paris polyphylla, Chem. Pharm. Bull. 30, 712-718.

[16] S. Rincón, R. E. Del Río, J. Sandoval-Ramírez, S. Meza-Reyes, S. Montiel-Smith, M. Fernández, N. Farfán and R. Santillan. (2006). A new route for the preparation of the 22,23-dioxocholestane side chain from diosgenin and its application to the stereocontrolled construction of the $22 R, 23 S$-diol function, Tetrahedron 62, 2594-2602.

[17] L. Tang, Z. Wang, H. Wu, A. Yokosuka and Y. Mimaki (2014). Steroidal glycosides from the underground parts of Dracaena thalioides and their cytotoxic activity, Phytochemistry 107, 102-110.

[18] J. Sandoval-Ramírez, A. Castro-Méndez, S. Meza-Reyes, F. Reyes-Vázquez, R. Santillán and N. Farfán (1999). Preparation of 22,26-epoxycholest-22-ene steroids. Novel transformation of the side chain in sapogenins. Tetrahedron Lett. 40, 5143-5146.

[19] I. Podolak, A. Galanty and D. Sobolewska (2010). Saponins as cytotoxic agents: a review. Phytochem. Rev. 9, 425-474.

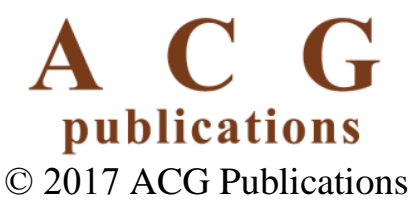

